\title{
TEORIA PARA O TRABALHO DE EXPANSÃO APLICADA ÀS BRISAS DO NORDESTE BRASILEIRO
}

\author{
CLÊNIA R. ALCÂNTARA ${ }^{1,2}$, ENIO P. SOUZA ${ }^{1}$ \\ ${ }^{1}$ Unidade Acadêmica de Ciências Atmosféricas - Universidade Federal de Campina Grande, \\ Campina Grande, PB. \\ ${ }^{2}$ Instituto de Astronomia, Geofísica e Ciências Atmosféricas/Universidade de São Paulo (IAG/USP), \\ São Paulo, SP. \\ clenia@model.iag.usp.br
}

Recebido Maio 2008 - Aceito Junho 2009

RESUMO

Neste artigo investiga-se numericamente a interação entre brisas marítima-terrestre e de valemontanha, que ocorrem no nordeste brasileiro devido à presença do Planalto da Borborema, aplicando o trabalho de expansão associado ao ramo inferior das circulações. Resultados da teoria da máquina térmica são comparados aos resultados 3D, obtidos com a versão brasileira do modelo RAMS. Os resultados indicaram que o efeito do contraste de temperatura no trabalho ligado às circulações (Wa), isoladamente, contribui para a formação de brisas marítimas mais intensas e de brisas terrestres menos intensas. Na realidade, o que se observa são brisas terrestres com intensidades iguais ou até maiores que das brisas marítimas. Assim, a contribuição da montanha para a intensidade das circulações de brisa no período noturno mostra-se extremamente não-linear. O trabalho de expansão que realmente está ligado às circulações, contribui em apenas $7 \%$ para o trabalho total. Dessa forma, a maior parte do trabalho total está associada ao trabalho de compressão que a atmosfera realiza para compensar a perda por resfriamento radiativo, e muito da energia disponibilizada para as circulações é gasta para vencer os processos dissipativos.

Palavras-chave: Brisas, Teoria Termodinâmica, Trabalho de Expansão, BRAMS.

\begin{abstract}
THEORY FOR EXPANSION WORK APPLIED TO BREEZES OF NORTHEAST OF BRAZIL

The purpose of this work is to further understand the interactions between sea-land breeze and valleymountain breeze that occur at the coast of Northeast Brazil due to the presence of the Borborema plateau, using the associated expansion work at the lower branch of these circulations. Results of the thermal machine theory are compared to the 3D results obtained from the Brazilian RAMS model version. The results indicate that the sole effect of temperature contrast on the work associated with circulations (Wa) is more intense sea breezes and weaker land breezes. Actually, one can observe land breezes whose intensities are equal or even larger than sea breezes. Thus, the mountain contribution for the intensity of the breeze circulations in the nighttime is extremely non-linear. The expansion work that is actually associated with these circulations contributes only with $7 \%$ for the total work. Consequently, the larger part of the total work is associated with the compression work performed by the atmosphere in order to compensate long-wave cooling and much of the energy available for the circulations is consumed to overcome dissipation.
\end{abstract}

Keywords: Breeze, Thermodynamic Theory, Expansion Work, BRAMS. 


\section{INTRODUÇÃO}

As circulações do tipo brisa foram e continuam sendo objeto de grande curiosidade científica. Apesar de serem chamadas circulações secundárias em relação à grande escala, são de grande importância para as áreas afetadas e podem contribuir significativamente para o total de precipitação e para o transporte de poluentes (Baummbach e Vogt, 1999), afetar a temperatura, a umidade, a nebulosidade (Planchon et al., 2006) e a produção agrícola (Bonnardot et al., 2005), por exemplo. Elas também se destacam ao interagir com outras circulações locais (Ohashi e Kida, 2002; Freitas, 2007) e com sistemas de escalas espaciais maiores, amplificando estes distúrbios de forma a produzirem, muitas vezes, tempestades severas.

Existem poucos estudos sobre o efeito da topografia na brisa marítima e o acoplamento entre as brisas marítima/terrestre e de vale-montanha utilizando uma topografia realista em um modelo numérico tridimensional (Federico et al., 2000; Miao et al., 2003; Porson et al., 2007; Perez-Landa et al., 2007; Alcântara e Souza, 2008). Isto porque, apenas recentemente, com o rápido avanço pelo qual vem passando as ciências computacionais, é que os modelos atmosféricos vêm sendo capazes de produzir simulações de alta resolução (em relação à escala das brisas) e reproduzir as condições atmosféricas com maior realismo. Assim, é possível continuar avançando no entendimento dos mais variados fenômenos atmosféricos. As circulações de brisa são bons fenômenos para se estudar a partir de tais experimentos, pois têm escalas espaciais e temporais pequenas, de forma que processos como turbulência e os efeitos orográficos não podem ser desprezados.

Federico et al. (2000), ao tentar estudar a importância relativa dos contrastes térmicos entre terra-mar e vale-montanha na determinação do desenvolvimento de circulações forçadas termicamente sobre a península montanhosa da Calábria, no sul da Itália, mostraram que a energia potencial disponível de mesoescala, liberada pela brisa marítima, aumenta com o aumento da profundidade da camada limite convectiva. Contudo, a energia potencial da brisa marítima diminui com o aumento de altitude, visto que a presença de uma montanha age como um obstáculo a sua penetração continente adentro. Já a energia potencial liberada pelo ar aquecido sobre a montanha aumenta com a profundidade da camada limite convectiva e com a sua altura. Com uma península montanhosa cercada pelo mar, a energia potencial total é a soma da energia potencial da brisa marítima com a energia potencial da brisa de vale.

Miao et al. (2003) examinaram uma região no leste da Espanha que possui topografia complexa. Eles mostraram que a topografia é um importante fator que influencia a circulação de brisa por alinhar a frente de brisa marítima com a linha da costa e localizar as zonas de convergência próximas à cadeia de montanhas. Quando a brisa marítima estava acoplada com a brisa de vale, ela ficou mais intensa e mais profunda.

Uma das teorias mais difundidas, que tem o intuito de estudar a energética da atmosfera, é a que aplica o conceito de máquina térmica. Os primeiros a aplicarem esta teoria à circulação geral da atmosfera foram Brunt (1926), Lettau (1954), Oort (1964) e Lorenz (1967). Eles mostraram que a eficiência termodinâmica da circulação geral é da ordem de $1 \%$. Lorenz (1967) argumentou que a determinação exata e a aplicação desta eficiência constituem o problema fundamental da energética da atmosfera.

Em seguida várias outras aplicações foram feitas em sistemas atmosféricos como furacões (Riehl, 1950; Emanuel, 1986), convecção (Rennó e Ingersoll, 1996), tornados e trombas d'água (Rennó e Bluestein, 2001), redemoinhos de poeira (Rennó et al., 1998) e circulações convectivas (Souza et al., 2000; Souza, 2004; Alcântara e Souza, 2008).

Souza (2004), ao utilizar aquele conceito, formulou uma teoria para o cálculo do trabalho de expansão associado aos ramos de um sistema de circulações induzidas por heterogeneidades. Souza (2004) confirmou o papel da dissipação como fonte extra de entropia para intensificação dos sistemas. Concluiu também, que o trabalho de expansão realizado próximo à superfície é compensado localmente pela compressão isobárica resultante do processo de resfriamento radiativo noturno. Por fim, concluiu que o trabalho de expansão associado à circulação é usado para vencer dissipação e o trabalho de compressão é usado para bombear calor de volta à superfície.

O objetivo deste artigo é mostrar o trabalho de expansão, que é derivado da aplicação da teoria da máquina térmica às circulações locais (Souza, 2004), associado ao ramo inferior das brisas marítima-terrestre e de vale-montanha, que acontecem no litoral do nordeste brasileiro devido à presença do Planalto da Borborema. Dessa forma, pretende-se entender um pouco mais da interação entre essas circulações e o papel da elevação em termos da energética das circulações.

\section{DADOS E METODOLOGIA}

\subsection{Teoria para o Trabalho de Expansão Aplicada às Circulações Locais}

O trabalho de expansão e compressão associado aos ramos de um sistema de circulação induzida por heterogeneidades à superfície foi derivado de Souza (1999) e Souza et al. (2000), e é mostrado detalhadamente por Souza (2004). Aqui é apresentada e discutida apenas a equação final, que é dada por:

$$
\mathrm{W}=\frac{\eta}{(1-\eta)}\left(\mathrm{c}_{\mathrm{p}} \Delta \mathrm{T}+\mathrm{L}_{\mathrm{\pi}} \Delta r\right)+\mathrm{R} \Delta \mathrm{T}+\frac{1}{(1-\eta \pi)} \mathrm{g} \Delta z
$$


em que $\mathrm{c}_{\mathrm{P}}$ é calor específico do ar seco à pressão constante, $\Delta T=T_{B}-T_{A}$ é a diferença de temperatura entre os pontos $\mathrm{A}$ e $\mathrm{B}$, $\mathrm{L}_{\mathrm{V}}$ é a o calor latente de vaporização, $\Delta_{\mathrm{r}}=\mathrm{r}_{\mathrm{B}}-\mathrm{r}_{\mathrm{A}}$ é a diferença de razão de mistura, $\mathrm{R}$ é a constante dos gases para o ar seco, $\mathrm{g}$ é a aceleração da gravidade e $\Delta \mathrm{z}=\mathrm{z}_{\mathrm{B}}-\mathrm{z}_{\mathrm{A}}$ é a diferença de altitude entre os pontos.

Para melhor se entender a contribuição da inclinação do terreno nos processos estudados, Souza (2004) dividiu a diferença de temperatura entre A e B (ver Figura 1) em duas partes, uma adiabática $\left(\Delta \mathrm{T}_{\mathrm{ad}}\right)$ e outra não-adiabática $\left(\Delta \mathrm{T}_{\mathrm{na}}\right)$. Logo,

$\Delta \mathrm{T}=\Delta \mathrm{T}_{\mathrm{ad}}+\Delta \mathrm{T}_{\mathrm{na}}=-\frac{g}{\mathrm{c}_{\mathrm{p}}} \boldsymbol{\Delta z}+\Delta \mathrm{T}_{\mathrm{n}} \Rightarrow \Delta \mathrm{T}_{\mathrm{na}}=\mathrm{T}_{\mathrm{B}}-\mathrm{T}_{\mathrm{A}}+\frac{\mathrm{g}}{\mathrm{c}_{\mathrm{p}}} \boldsymbol{\Delta z}$

Então, substituindo $\Delta \mathrm{T}_{\text {na }}$ na Equação 1 obtém-se:

$$
W=\frac{\gamma \eta}{(1-\gamma \eta)}\left(c_{p} \Delta T_{n a}+L_{\nu} \Delta r\right)+R \Delta T_{n a}+\frac{1}{k} g \Delta z
$$

em que é $k=\mathrm{c}_{\mathrm{P}} / \mathrm{c}_{\mathrm{V}}$ e $\mathrm{c}_{\mathrm{V}}$ é o calor específico do ar seco à volume constante.

$\mathrm{O}$ primeiro termo da Equação 3 (Wa) refere-se à quantidade de trabalho extra que é realizado a partir do calor absorvido próximo à superfície (reservatório quente) devido ao aquecimento resultante da absorção de calor e da dissipação de energia. Este é, de fato, o trabalho útil que será usado pela circulação, chamado de trabalho de escoamento, por tratar-se de um sistema aberto. $\mathrm{O}$ segundo termo $(\mathrm{Wb})$ refere-se à absorção de calor latente, quando há condensação ao longo da circulação. Aqui, este termo será desprezado já que não houve condensação ao longo da circulação de brisa, conforme descrito por Alcântara e Souza (2008). O terceiro termo (Wc) é o trabalho isobárico que existe mesmo não havendo circulações convectivas. Por fim, o quarto termo $(\mathrm{Wd})$ representa a expansão devido à diminuição de pressão hidrostática quando a parcela aumenta de altitude.

$\mathrm{Na}$ equação 3, o termo referente à umidade, que é importante no caso de sistemas precipitantes saturados, será omitido nos cálculos realizados posteriormente, pois é considerado que nas simulações não houve condensação ao longo da circulação de brisa.

A eficiência termodinâmica $\eta$ é obtida a partir da seguinte equação (Souza et al. 2000):

$$
\eta=\frac{\mathrm{B} Z}{\mathrm{c}_{\mathrm{p}} \mathrm{T}_{\mathrm{h}}}
$$

em que $T_{h}$ é a temperatura da região onde calor é absorvido (próximo à superfície), e $\mathrm{Z}$ é a profundidade da camada limite turbulenta.
Quanto à fração de dissipação que ocorre próximo à superfície $\gamma$, ela será considerada igual a 0,60 , de acordo com experimentos realizados por Alcântara e Souza (2008) que mostraram ser este valor o mais adequado para as situações aqui estudadas. Assim, 60 \% de toda dissipação ocorre próxima à superfície.

As variáveis envolvidas nos cálculos da equação 3, como a temperatura e a razão de mistura, foram retiradas das simulações que serão detalhadas a seguir.

\subsection{Características das Simulações e Cálculos}

As simulações e os experimentos realizados são os mesmos utilizados por Alcântara e Souza (2008). Aqui será feita apenas uma breve descrição.

Foram processadas duas simulações utilizando o Brazilian Regional Atmospheric Modeling System (BRAMS), as quais tiveram como condição inicial as análises do Modelo Global do Centro de Previsão de Tempo e Estudos Climáticos (CPTEC) do Instituto Nacional de Pesquisas Espaciais (INPE), com duas grades aninhadas, uma com $20 \mathrm{~km}$ e outra com $5 \mathrm{~km}$, e início a 00 UTC do dia 01 de novembro de 2003 e final a 00 UTC do dia 13 de novembro de 2003. A primeira simulação foi feita com a topografia realística da região em estudo (Topo) e a segunda, sem topografia (STopo).

As parametrizações utilizadas foram: Chen e Cotton (1983), para radiação de onda curta e onda longa, o LEAF-3 com modelo solo/vegetação, e para a difusão turbulenta, deformação anisotrópica segundo Smagorinsky (1963). A microfísica (Walko et al. 1995) foi ativada no nível 2 e a parametrização de cúmulos não foi ativada. Estas últimas duas opções foram adotadas para evitar que o modelo produzisse chuva, o que não ocorreu durante o período. Desta forma, teve-se o cuidado de se produzir simulações não-saturadas, e assim, o termo ligado à umidade na Equação 3 pôde ser desprezado. Além disso, o que permite que esse termo de liberação de calor latente seja desprezado é a falta de processos de condensação, principalmente ao longo da circulação.

Todos os cálculos e as análises que seguem, foram feitos sobre as latitudes de $7^{\circ} \mathrm{S}$ e $8^{\circ} \mathrm{S}$. Após verificar-se o comprimento horizontal das brisas, tomou-se como ponto de referência sobre o continente, a longitude de $35,25^{\circ} \mathrm{W}$ (ponto B) e sobre o oceano, a longitude de $34,75^{\circ} \mathrm{W}$ (ponto A) (ver Figura 1). Assim, a diferença de altitude entre os pontos é de aproximadamente $100 \mathrm{~m}$ sobre a latitude de $7^{\circ} \mathrm{S}$ e $140 \mathrm{~m}$ sobre $8^{\circ} \mathrm{S}$. O resumo dos experimentos encontra-se na Tabela 1 .

Para se calcular a eficiência termodinâmica (Equação 4) nas simulações, $\mathrm{T}_{\mathrm{h}}$ foi considerada a temperatura do primeiro nível atmosférico disponível (10m acima da superfície) e, juntamente com Z, é obtida em cada ponto de grade entre as 


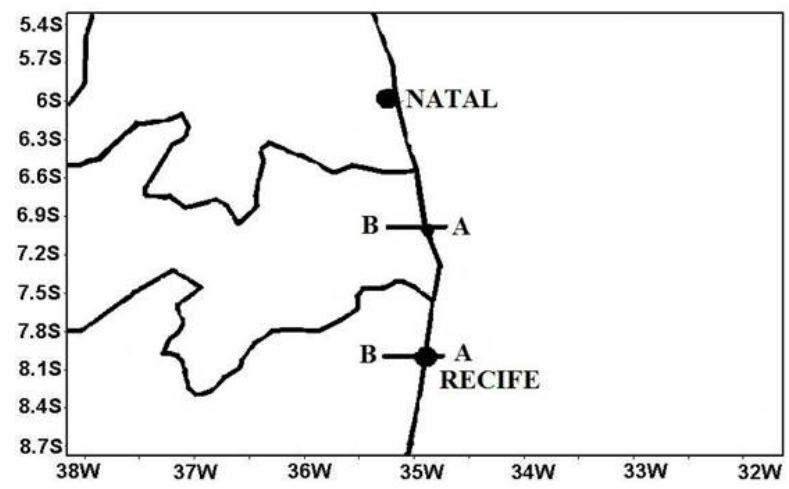

Figura 1 - Área compreendida pela grade interna utilizada nas simulações, com as indicações dos pontos $\mathrm{A}\left(34,75^{\circ} \mathrm{W}\right)$ e $\mathrm{B}\left(35,25^{\circ} \mathrm{W}\right)$ ao longo das latitudes de $7^{\circ} \mathrm{S}$ e $8^{\circ} \mathrm{S}$ e das cidades de Natal - RN, João Pessoa - PB e Recife - PE.

Tabela 1 - Experimentos realizados e suas descrições.

\begin{tabular}{|l|l|}
\hline EXPERIMENTO & \multicolumn{1}{|c|}{ DESCRIÇÃO } \\
\hline Topo7S & Simulação com topografia e resultados analisados sobre a latitude $7^{\circ} \mathrm{S}$ \\
\hline Topo8S & Simulação com topografia e resultados analisados sobre a latitude $8^{\circ} \mathrm{S}$ \\
\hline STopo7S & Simulação com topografia e resultados analisados sobre a latitude $7^{\circ} \mathrm{S}$ \\
\hline
\end{tabular}

longitudes de $35,25^{\circ} \mathrm{W}$ e $34,75^{\circ} \mathrm{W}$. Dessa forma, a eficiência utilizada nos cálculos que seguem é a média das eficiências observadas em cada ponto de grade ao longo de uma latitude e entre as longitudes de $35,25^{\circ} \mathrm{W}$ e $34,75^{\circ} \mathrm{W}$.

O cálculo do trabalho de expansão foi feito a partir da integração numérica entre os pontos A e B de $(p d \alpha)$. Já o termo Wa foi obtido pela integração numérica entre os pontos $\mathrm{A}$ e $\mathrm{B}$ de $(-\alpha d p)$. O volume específico, em cada ponto, é calculado usando a temperatura virtual.

\subsection{Dados Observados}

Foram utilizados valores de Convective Available Potential Energy (CAPE) calculados a partir de sondagens realizadas no período simulado entre 03 e 13 de novembro de 2003 para as cidades de Recife - PE $\left(8,05^{\circ} \mathrm{S} ; 34,91^{\circ} \mathrm{W}\right)$ e Natal - RN $\left(5,91^{\circ} \mathrm{S} ; 35,25^{\circ} \mathrm{W}\right)$ (Ver Figura 1), no horário das 12 UTC. Essas sondagens são provenientes de duas fontes, do CPTEC - INPE e do Departamento de Ciências Atmosféricas da Universidade de Wyoming - EUA (ver Referências Bibliográficas).

\section{RESULTADOS E DISCUSSÃO}

A validação das simulações foi apresentada em Alcântara e Souza (2008). Naquela ocasião, o experimento Topo7S foi comparado aos dados observados em João Pessoa $\left(7,08^{\circ} \mathrm{S}\right.$; $\left.34,83^{\circ} \mathrm{W}\right)$. Pode-se considerar que a simulação está em boa concordância com os dados observados.

Aqui serão apresentadas comparações entre o trabalho obtido diretamente das simulações e o trabalho calculado a partir da teoria, a fim de se entender um pouco mais a respeito do papel da elevação de terrenos em termos da energética das circulações.

Nas Figuras 2 e 3 observa-se o trabalho total obtido das simulações (Simulado) e o trabalho total calculado através da teoria (Teoria) para Topo7S e STopo7S, respectivamente. Nelas, percebe-se que a variação temporal do trabalho total apresenta um padrão de oscilação associado ao ciclo diurno. Para Topo7S seus valores ficaram entre $319 \mathrm{~J} \mathrm{Kg}^{-1}$ e $2460 \mathrm{~J}$ $\mathrm{Kg}^{-1}$. Para Topo8S, o trabalho total mostrou-se um pouco maior em média, variando seus valores durante o dia entre $661 \mathrm{~J} \mathrm{Kg}^{-1}$ e $2877 \mathrm{~J} \mathrm{Kg}^{-1}$ (figura não mostrada). E para STopo7S, esses valores foram bem menores ficando entre $-267 \mathrm{~J} \mathrm{Kg}^{-1}$ e $1700 \mathrm{~J}$ $\mathrm{Kg}^{-1}$. Assim, o trabalho de expansão realizado pelas circulações é maior à medida que se aumenta a diferença de altitude entre os pontos $\mathrm{A}$ e $\mathrm{B}$.

Na Figura 4 tem-se uma comparação entre o trabalho observado no experimento STopo7S (W STopo7S) e os valores de CAPE, obtidos a partir das sondagens fornecidas pelo CPTEC - INPE (CAPE_CPTEC) e pela Universidade de Wyoming - EUA (CAPE_UW) para Recife - PE e Natal 
- RN. A comparação da CAPE observada com o trabalho de STopo7S, é justificada pelo fato dessas cidades estarem ao nível médio do mar, e portanto, não sofrerem o efeito da diferença de altitude. Note-se que o trabalho de expansão é da mesma ordem de magnitude dos valores de CAPE. Isto confirma que a maior parte do trabalho de expansão da atmosfera ocorre pela absorção de calor próximo à superfície, conforme postulado por Rennó e Ingersoll (1996).
O termo mais importante para as circulações locais na Equação 3 é o primeiro (Wa), que está ligado às circulações de brisa, pois representa a única contribuição associada à conversão de entropia absorvida ao longo do ramo inferior da circulação e que é convertida em energia cinética da circulação. As figuras a seguir mostram este primeiro termo da Equação 3 [Teoria] comparando-o com o trabalho obtido nas simulações (Simulado) para Topo7S (Figura 5) e Stopo7S (Figura 6).

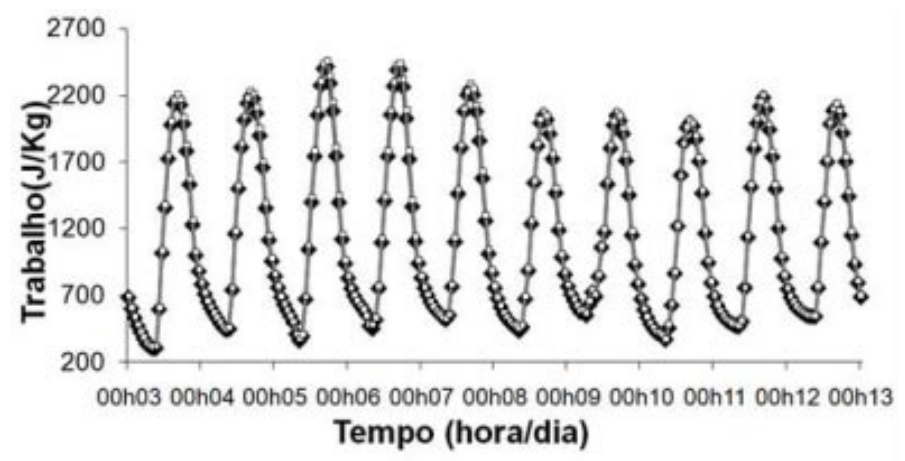

Teoria - Simulado

Figura 2 - Trabalho total calculado pela teoria (Teoria) e obtido das simulações (Simulado) para Topo7S.

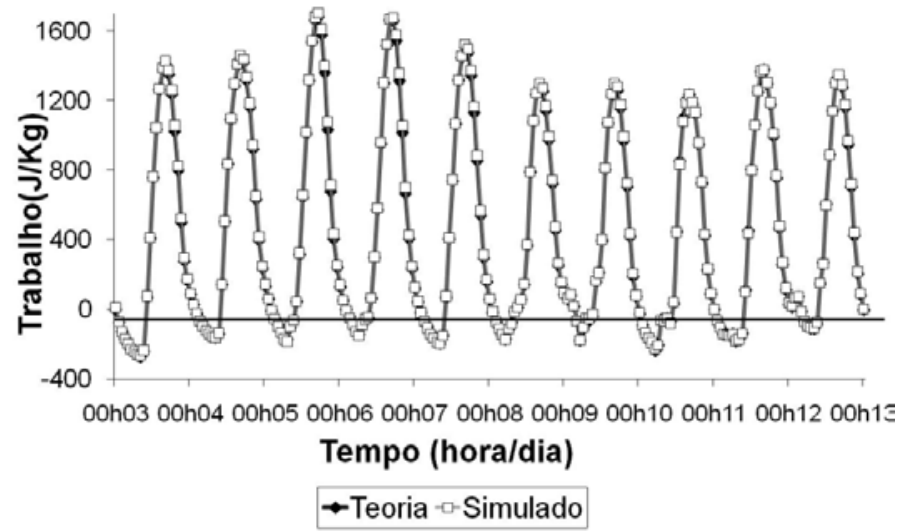

Figura 3 - Trabalho total calculado pela teoria (Teoria) e obtido das simulações (Simulado) para STopo7S.

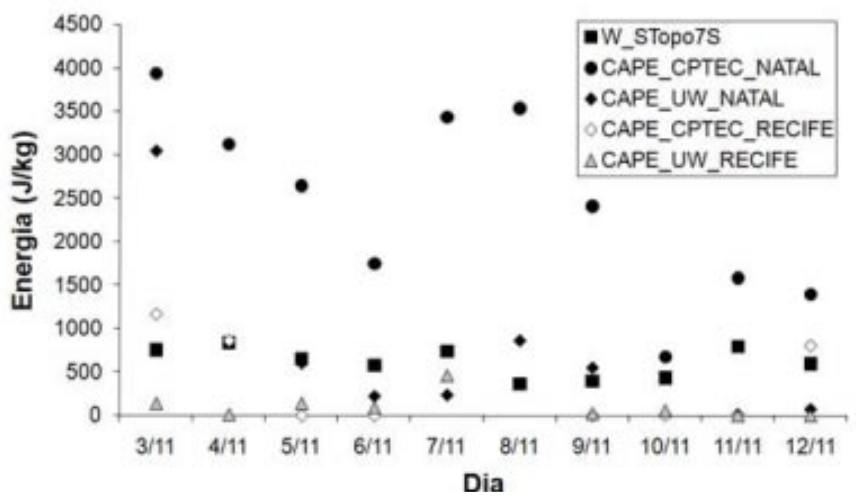

Figura 4 - Trabalho total obtido do experimento STopo7S (W_STopo7S), CAPE obtida pela Universidade Wyoming para Natal (CAPE UW NATAL) e Recife (CAPE_UW_RECIFE) e CAPE obtida pelo CPTEC para Natal (CAPE_CPTEC_NATAL) e Recife (CAPE_CPTEC_RECIFE). Todas às 12 UTC. 
Observa-se que para Topo7S os valores de Wa variam entre $-15 \mathrm{~J} \mathrm{Kg}^{-1}$ e $114 \mathrm{~J} \mathrm{Kg}^{-1}$. Para Topo8S esses valores encontram-se entre $-9 \mathrm{~J} \mathrm{Kg}^{-1}$ e $124 \mathrm{~J} \mathrm{Kg}^{-1}$ (figura não mostrada). E por fim, para STopo7S, tem-se que o trabalho encontra-se entre $-12 \mathrm{~J} \mathrm{Kg}^{-1}$ e $104 \mathrm{~J} \mathrm{Kg}^{-1}$. Da mesma forma que para o trabalho de expansão total, esses termos também aumentam à medida que se aumenta a diferença de altitude entre os pontos, e isso é devido à contribuição de $\Delta \mathrm{T}_{\text {na }}$ no cálculo realizado. Note-se que os valores negativos do trabalho ocorridos durante a noite, não significam trabalho de compressão. Como o cálculo é feito tomando-se a diferença de temperatura entre continente e oceano, um valor negativo indica que o ar se expande à medida que a circulação é dirigida da terra para o mar.

Já nas Figuras 7 e 8, pode-se ver o trabalho ligado às circulações (Wa) para os três experimentos, porém, na primeira figura tem-se esse trabalho calculado com $\Delta \mathrm{T}_{\text {na }}$ que evidencia o efeito da topografia para as latitudes de $7^{\circ} \mathrm{S}$ (Topo7S) e $8^{\circ} \mathrm{S}$ (Topo8S). Na figura seguinte, foi utilizado $\Delta \mathrm{T}$ para Topo $7 \mathrm{~S}$ e Topo8S desconsiderando, assim, o efeito da topografia.
Na Figura 7, observa-se que os três experimentos mantêm-se muito próximos, a não ser para os dois primeiros casos de brisa marítima do período simulado, no qual o experimento Topo8S obteve maiores valores de trabalho. Isso ressalta a importância da topografia para incrementar a circulação.

Na Figura 8, observam-se algumas diferenças entre os experimentos. Para os casos ligados às brisas marítimas, STopo7S obteve os maiores valores de trabalho. Para os casos de brisa terrestre, nos quais se observam as maiores diferenças entre os experimentos, o maior trabalho em módulo é observado para Topo8S e os menores valores em módulo desse trabalho de expansão, no sentido terra-mar, são encontrados em STopo7S. Isto indica que as diferenças apresentadas anteriormente são devidas quase que totalmente às diferenças na topografia da região. Os resultados apresentados aqui estão coerentes com aqueles observados em Alcântara e Souza (2008) [ver Figura 6 daquele artigo], que mostra a diferença de temperatura entre o continente e o oceano.

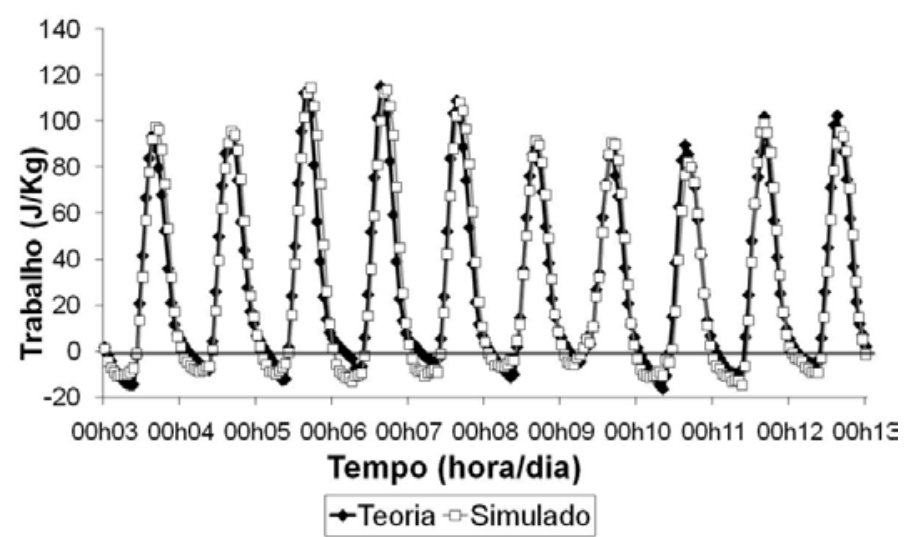

Figura 5 - Trabalho ligado à circulação (Wa) calculado pela teoria (Teoria) e obtido da simulação (Simulado) para Topo7S.

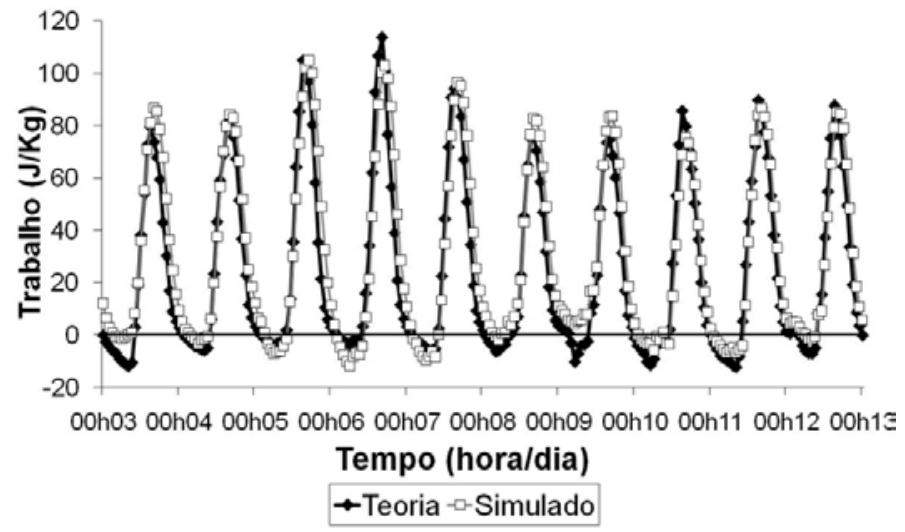

Figura 6 - Trabalho ligado à circulação (Wa) calculado pela teoria (Teoria) e obtido da simulação (Simulado) para STopo7S. 
Comparando as Figuras 7 e 8, percebe-se que o efeito exercido pela topografia no trabalho de expansão ligado às brisas terrestres, é de diminuição de seus valores. Para os casos de brisa marítima, observa-se um aumento nos valores de trabalho. Isso pode ser visto mais detalhadamente para os experimentos Topo7S e Topo8S nas Figuras 9 e 10, nas quais se tem que para $7^{\circ} \mathrm{S}$, o trabalho diminui cerca de $10 \mathrm{~J} \mathrm{Kg}^{-1}$ e para $8^{\circ} \mathrm{S}$, cerca de $20 \mathrm{~J} \mathrm{Kg}^{-1}$, nos casos de brisa terrestre, e aumenta aproximadamente o dobro desses valores para os casos de brisa marítima em ambos os experimentos.

Os resultados das Figuras 7 e 8 sugerem um papel diferente para a inclinação do terreno, em relação ao seu efeito

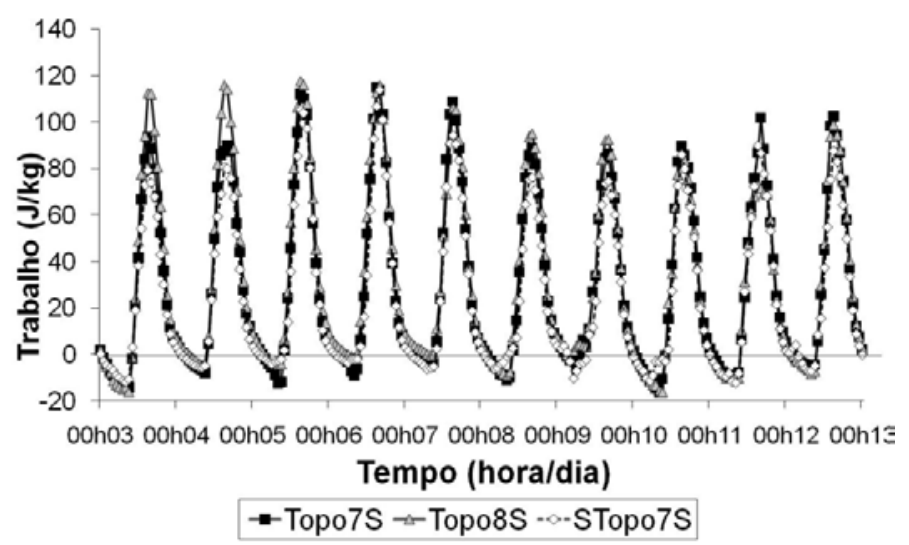

Figura 7 - Trabalho ligado à circulação (Wa) para Topo7S e Topo8S, com o efeito da topografia, e para STopo7S.

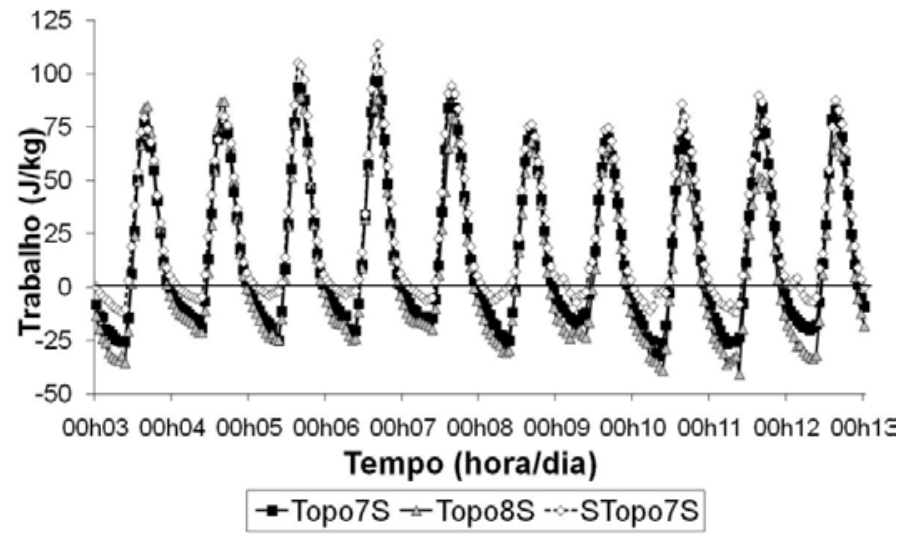

Figura 8 - Trabalho ligado à circulação (Wa) para Topo7S e Topo8S, sem o efeito da topografia, e para STopo7S.

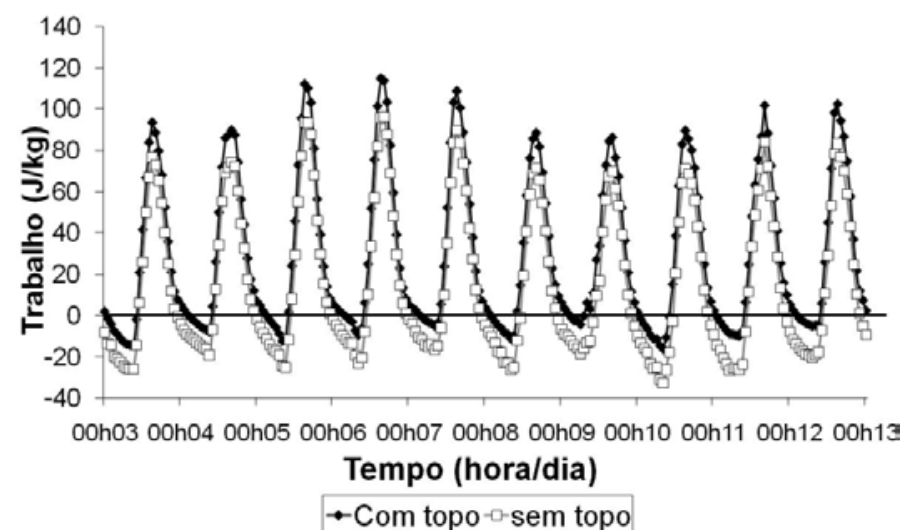

Figura 9 - Trabalho ligado à circulação (Wa) com e sem o efeito da topografia para Topo7S. 
nas brisas diurna e noturna. Durante o dia, à medida que a combinação brisa marítima + brisa de vale força o escoamento montanha acima, o ar recebe um fluxo de calor extra, caso se resfrie a uma taxa correspondente a uma expansão adiabática. Como o solo montanha acima tende a estar mais quente do que o ar logo acima, acontece um fluxo de calor extra que aumenta a intensidade da brisa (Souza et al., 2000).

No caso da brisa noturna, quando o ar desce montanha abaixo, devido ao aquecimento adiabático de compressão, há um fluxo de calor da parcela para o solo que pode ser visto na Figura 11, que mostra o fluxo de calor sensível simulado entre a 01 UTC e as 09 UTC do dia 03 de novembro de 2003 para Topo7S. Isso é observado em todos os períodos noturnos de todos os dias simulados (ver Figura 17). Como no presente caso a brisa não aparece isoladamente, isto é, o escoamento continua a ser montanha acima (devido aos ventos de leste, associados à Alta Subtropical), o que acontece é que, à medida que sobe, $o$ ar perde calor para a montanha. Isso faz com que o trabalho no sentido mar-terra seja negativo.

Assim, era de se esperar que a intensidade das circulações de brisa terrestre fosse menor que a intensidade das circulações de brisa marítima. Porém, um outro efeito pode ser adicionado a este explicado acima. À medida que a parcela desce a montanha e é comprimida, ela fica cada vez mais densa e por isso acelera, contribuindo para que a circulação seja mais intensa que o esperado. Isso implica em um grau de não-linearidade, que não consegue ser capturado de forma simples pela teoria.

Ainda em relação ao trabalho ligado às circulações (Wa), pode-se destacar que seus máximos ligados às brisas marítimas ocorrem em torno das 16 UTC, ou seja, às $13 \mathrm{HL}$, que é o horário típico em que se observa o desenvolvimento de nuvens convectivas profundas na região de estudo (no período

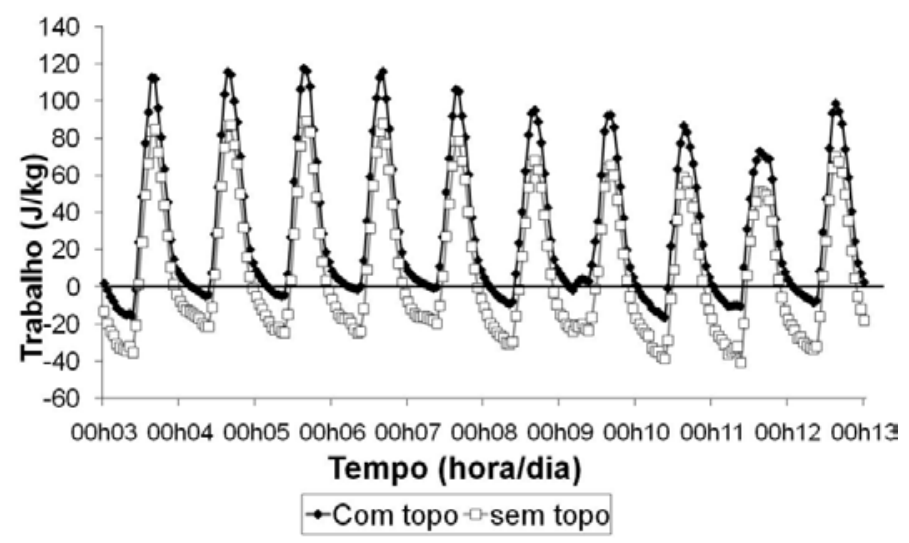

Figura 10 - Trabalho ligado à circulação (Wa) com e sem o efeito da topografia para Topo8S.

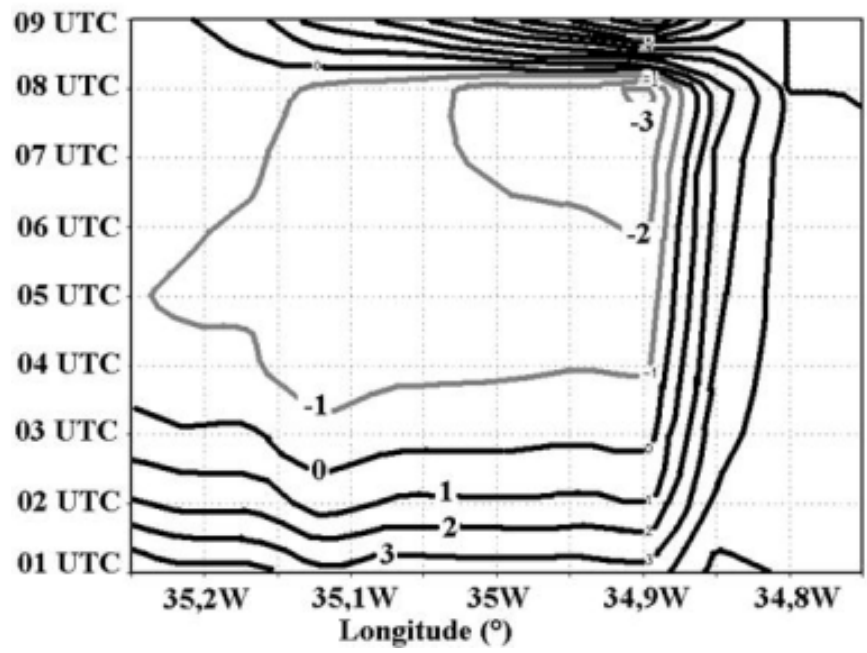

Figura 11 - Fluxo de calor sensível simulado entre a 01 UTC e as 09 UTC do dia 03 de novembro de 2003 para Topo7S. 
chuvoso). Além disso, este trabalho possui a mesma ordem de magnitude da energia de inibição convectiva (CINE) que uma parcela teria de "vencer" para chegar ao seu nível de convecção livre (Williams e Rennó, 1993). Assim, pode-se especular aqui que esta energia disponibilizada para a circulação, e indicada por
Wa, pode ser um mecanismo que leve ao disparo da convecção sobre o continente.

Nas três figuras que seguem (Figuras 12, 13 e 14), pode-se ver a contribuição individual de cada um dos termos da Equação 3 para o trabalho total nos três experimentos. Lembra-

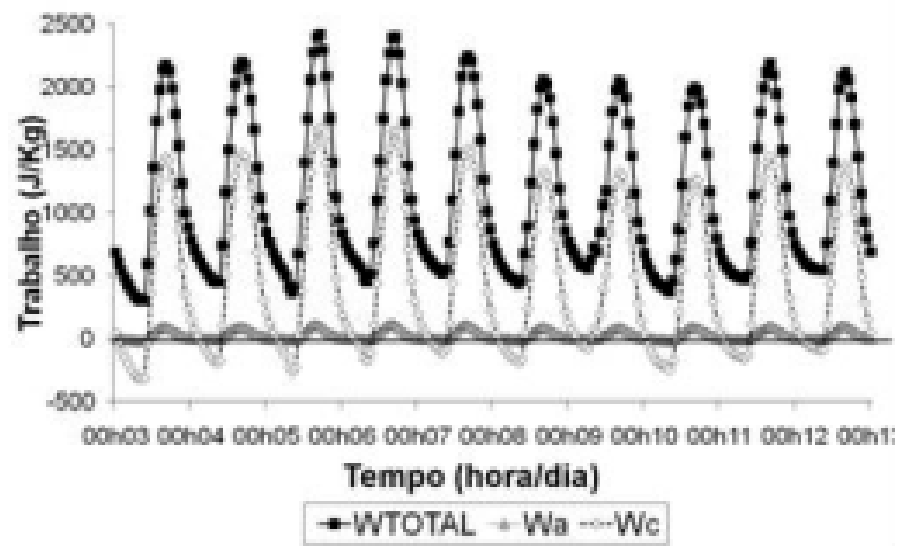

Figura 12 - Contribuição do primeiro (Wa) e terceiro (Wc) termos para o trabalho de expansão total (WTOTAL) calculado pela teoria para Topo $7 \mathrm{~S}$.

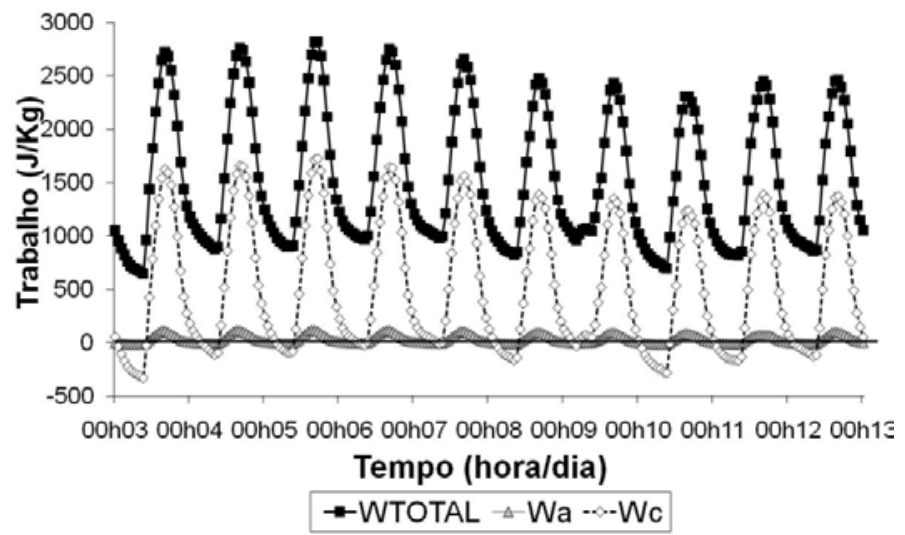

Figura 13 - Contribuição do primeiro (Wa) e terceiro (Wc) termos para o trabalho de expansão total (WTOTAL) calculado pela teoria para Topo8S.

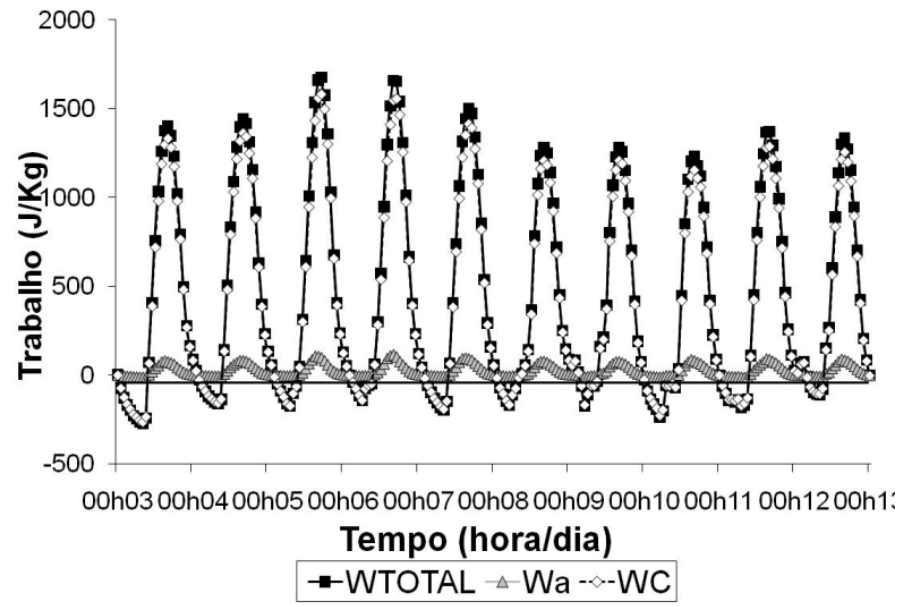

Figura 14 - Contribuição dos primeiro (Wa) e terceiro (Wc) termos para o trabalho de expansão total (WTOTAL) calculado pela teoria para STopo7S. 
se aqui que o primeiro termo (Wa), discutido anteriormente, é devido ao calor absorvido ao longo do ramo inferior da circulação de brisa, o segundo termo foi omitido dos cálculos, pois não se considera condensação ao longo da circulação, o terceiro termo (Wc) é o trabalho isobárico, que representa a expansão que ocorre quando o ar absorve calor em virtude do seu contato com a superfície e, por fim, o último termo (Wd) é a expansão devida à diminuição de pressão hidrostática quando a parcela aumenta de altitude.

O termo Wd é constante em todos os experimentos, pois depende apenas da diferença de altitude entre os pontos. Para Topo7S, Wd foi de 635,29 $\mathrm{J} \mathrm{Kg}^{-1}$. Para Topo8S foi de 992,86 $\mathrm{J} \mathrm{Kg}^{-1}$. E para STopo7S foi de $0 \mathrm{~J} \mathrm{Kg}^{-1}$. Percebe-se que a contribuição de Wa para o trabalho total é muito pequena comparada com os demais termos, mesmo para o experimento STopo7S no qual o termo Wd é zero. Segundo Souza (2004), esse termo contribui com apenas $10 \%$ do trabalho total. Aqui se tem que essa contribuição foi de no máximo 7\%. Logo, a maior parte do trabalho de expansão não está associada às circulações locais e sim, a uma compensação resultante dos processos radiativos pela qual a atmosfera realiza trabalho de compressão. Para os experimentos com topografia, outra parte desse trabalho total ainda está diretamente ligada à expansão que ocorre quando uma parcela de ar sobe um terreno inclinado.

A Figura 15 mostra a comparação entre o trabalho total simulado e a perturbação simulada da componente zonal do vento. Por sua vez, a Figura 16 mostra o trabalho total simulado e o fluxo de calor sensível, ambas para o experimento Topo7S. Percebe-se que o trabalho máximo ocorre antes de se observar a máxima intensidade do vento e que no horário que se observa esse trabalho máximo, também se observa o máximo do fluxo de calor sensível, indicando que a turbulência também é máxima

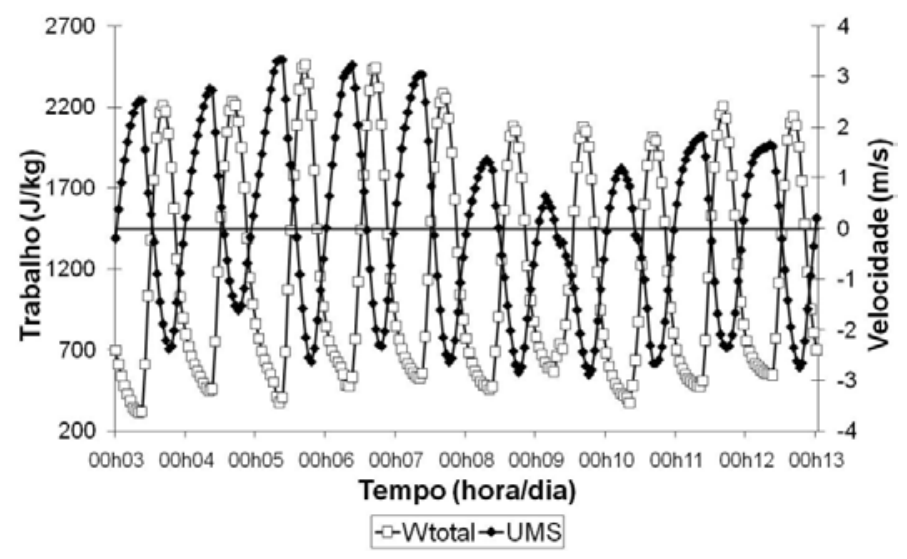

Figura 15 - Trabalho total de expansão simulado (Wtotal) e perturbação simulada da componente zonal do vento (UMS) para Topo7S.

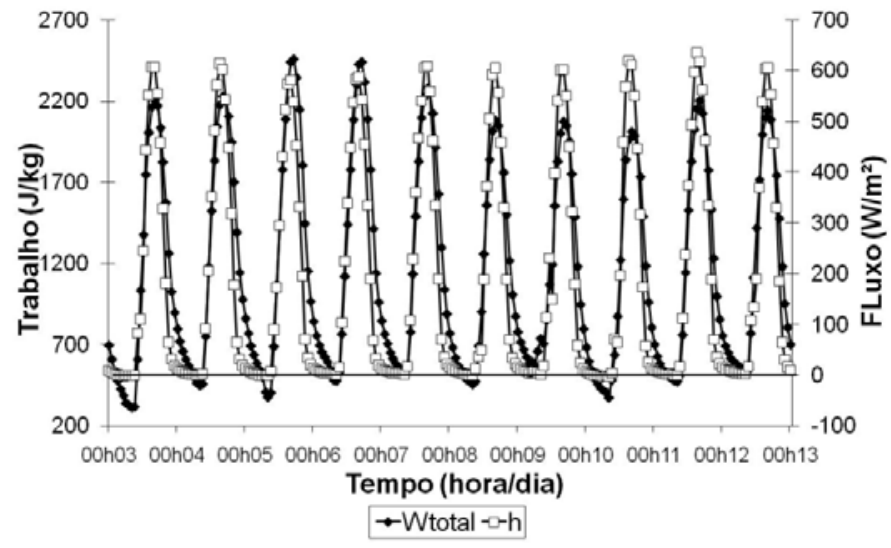

Figura 16 - Trabalho total de expansão simulado (Wtotal) e fluxo de calor sensível (h) para Topo7S. 


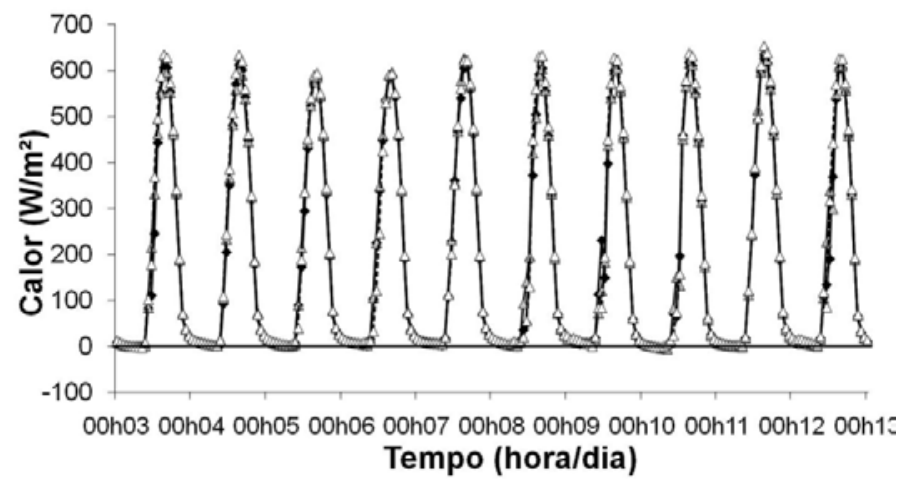

-Topo7S $\leadsto-$ Topo8s $\leadsto$ STopo7S

Figura 17 - Fluxo de calor sensível para Topo7S, Topo8S e STopo7S.

Tabela 2 - Parâmetros estatísticos para análise do ajuste entre os dados, em que r é o coeficiente de correlação, RMSE é a raiz do erro médio quadrático, $(\mathrm{RMSE})_{\mathrm{BIAS}}$ é RMSE retirado um bias constante, $\sigma_{\mathrm{SIM}} / \sigma_{\mathrm{TEO}}$ são os desvios-padrão dos dados das simulações e teóricos, respectivamente.

\begin{tabular}{|c|c|c|c|c|}
\hline Variáveis Comparadas & $\mathbf{r}$ & $\mathbf{R M S E}$ & (RMSE) $_{\text {BIAS }}$ & $\underline{\mathbf{S}}_{\mathbf{S I M}} \underline{\mathbf{S}}_{\text {TEO }}$ \\
\hline W teórico (Topo7s) X simulado & 0,99 & 26,82 & 15,12 & $638,41 / 626,88$ \\
\hline W teórico (Topo8s) X simulado & 0,99 & 32,85 & 17,30 & $652,60 / 637,25$ \\
\hline W teórico (Topo7s) X simulado & 0,99 & 13,29 & 11,63 & $588,04 / 581,96$ \\
\hline
\end{tabular}

naquele instante. Assim, muito da energia que é convertida e disponibilizada para a circulação, é utilizada para vencer a dissipação imposta pelos processos turbulentos. Por isso, a intensidade máxima da circulação tende a ser observada em um período no qual a quantidade de energia disponibilizada é menor, mas que é utilizada para vencer menos dissipação.

Na Figura 17, tem-se a variação temporal do fluxo de calor sensível $\left(\mathrm{Wm}^{-2}\right)$ observada durante o período mostrado para os três experimentos em questão. Percebe-se que não há distinção entre eles, o que pode indicar que os processos turbulentos dos três experimentos tiveram intensidades similares. Os valores dos fluxos mostram-se entre $-10 \mathrm{Wm}^{-2} \mathrm{e}$ $650 \mathrm{Wm}^{-2}$ e também seguem um ciclo diurno.

Percebe-se que a teoria seguiu muito bem a tendência dos dados simulados. Assim, pode-se considerar que a teoria está em boa concordância com as simulações, como pode ser confirmado pelas informações estatísticas mostradas na Tabela 2 , em que se observam os coeficientes de correlação (r), raiz do erro médio quadrático (RMSE), RMSE depois de removido o erro sistemático (bias) constante [(RMSE)BIAS] e os devios- padrão dos dados observados (sOBS) e dos dados simulados (s). Estes últimos parâmetros foram calculados segundo Pielke (2002).

Para as correlações (r) mostradas neste trabalho foi aplicado um teste de significância através da distribuição $t$ de Student (Bussab e Morettin, 1987). Assim, para o nível de significância de $1 \%$, as correlações são estatisticamente significativas se maiores que 0,16 .

\section{CONCLUSÕES}

Foi apresentada neste artigo uma aplicação de teoria termodinâmica da máquina térmica que permite quantificar e caracterizar os efeitos combinados do contraste terra-mar com a inclinação do terreno na intensidade das circulações de brisa, através do cálculo do trabalho de expansão e compressão no ramo inferior das circulações de brisa que ocorrem no litoral do nordeste brasileiro.

O efeito do contraste de temperatura, isoladamente, contribui para a formação de brisas marítimas mais intensas e 
de brisas terrestres menos intensas. O mesmo efeito se tem no trabalho ligado às circulações (Wa). Assim, com a topografia, os valores de trabalho mostraram-se menores para os casos de brisa terrestre e maiores para os casos de brisa marítima. Porém, a intensidade das brisas terrestres mostrou-se igual ou até maior que a intensidade das brisas marítimas. Logo, a contribuição da montanha para a intensidade das circulações de brisa no período noturno mostra-se extremamente não-linear. Por um lado, uma parcela que desce a inclinação pode estar mais aquecida, devido ao aquecimento adiabático de compressão, que a superfície imediatamente abaixo provocando um fluxo negativo de calor sensível, o que tenderia a diminuir sua intensidade. Por outro lado, à medida que essa parcela desce a montanha, fica mais densa e tende a acelerar.

O trabalho de expansão que realmente está ligado às circulações, contribui em apenas 7\% para o trabalho total de expansão. Assim, a maior parte deste trabalho está associada ao trabalho de compressão que a atmosfera realiza para compensar a perda por resfriamento radiativo. Independente da inclinação, esse trabalho é da ordem de $100 \mathrm{~J} \mathrm{Kg}^{-1}$, o que ressalta o papel das brisas como mecanismo de favorecimento na formação das nuvens convectivas profundas sobre o continente.

Muita da energia disponibilizada para as circulações é gasta para vencer os processos dissipativos, principalmente no período diurno, quando esses processos são realmente efetivos. Isso explica a defasagem encontrada entre o máximo do trabalho de expansão e o máximo nas intensidades das circulações. Este também pode ser um fator que contribui para que as brisas marítimas não sejam muito mais intensas que as brisas terrestres, posto que à noite não há turbulência térmica $\mathrm{e}$, portanto, tende a haver, efetivamente, mais energia disponível para as circulações.

Por fim, percebe-se que a teoria consegue prever com boa aproximação os resultados observados nas simulações. Em particular, para os casos de brisa marítima quando estas se apresentam bem definidas e relativamente intensas.

\section{AGRADECIMENTOS}

Os autores agradecem ao $\mathrm{CNPq}$ pelo suporte à realização deste trabalho.

\section{REFERÊNCIAS BIBLIOGRÁFICAS}

ALCÂNTARA, C. R.; SOUZA, E. P.; Uma teoria termodinâmica para brisas: teste utilizando simulações numéricas. Revista Brasileira de Meteorologia, 23, 1-11, 2008.

BAUMBACH, G.; VOGT, U.; Experimental determination of the effect of mountain-valley breeze circulation on air pollution in the vicinity of Freiburg. Atmospheric
Environment, 33, 4019-4027, 1999.

BONNARDOT, V.; PLANCHON, O; CAUTENET, S.; Sea breeze development under an offshore synoptic wind in the South-Western Cape and implications for the Stellenbosch wine-producing area. Theor. Appl. Climatol., 81, 203-218, 2005.

BRUNT, D.; Energy in the earth's atmosphere. Philos. Mag., v.7, p. 523-532, 1926.

BUSSAB, W. O.; MORETTIN, P. A.; Estatística Básica, 4ed. Atual Editora, 1987. p. 288 - 291.

CHEN, C.; COTTON, W. R.; A one-dimensional simulation of the stratocumulus capped mixed layer. Bound.-Lay. Met., v.25, p. 289-321, 1983.

CPTEC - Centro de previsão de tempo e estudos climáticos - www.cptec.inpe.br/dados_observados/.

Departamento de Ciências Atmosféricas da Universidade de Wyoming - EUA - http://weather.uwyo.edu/upperair/ sounding.html.

EMANUEL, K. A.; An air-sea interaction theory for tropical cyclones. Part I: steady-state. J. Atmos. Sci. v.43, p. 585604. 1986.

FEDERICO, S.; DALU, G. A.; BELLECCI, C.; COLACINO, M.; Mesoscale energetics and flows induced by sea-land and mountain-valley contrasts. Ann. Geophysicae, v.18, p.235-246. 2000.

FREITAS, E. D.; ROZOFF, C. M.; COTTON, W. R.; SILVA DIAS, P. L.; Interactions of an urban heat island and seabreeze circulations during winter over the metropolitan area of São Paulo, Brazil. Boundary-Layer Meteorol, 122, 1, 43-65(23), 2007.

LETTAU, H.; A study of the mass, momentum and energy budget of the atmosphere. Arch. Meteor. Geophys. Bioklimatol., A7, p. 133-157, 1954.

LORENZ, E. N.; The nature and theory of the general circulation of the atmosphere. World Meteorological Organization, p.161, 1967.

MIAO, J.-F; KROON, L. J. M.; VILÁ-GUERAU DE ARELlANO, J.; HOLTSLAG, A. A. M.; Impacts of topography and land degradation on the sea breeze over eastern Spain. Meteorol Atmos Phys, v.84, p.157-170, 2003.

OHASHI, Y.; KIDA, H.; Local Circulations Developed in the Vicinity of Both Coastal and Inland Urban Areas: A Numerical Study with a Mesoscale Atmospheric Model. Journal of Applied Meteorology, 41, 30-45, 2002.

OORT, A. H.; On estimates of the atmosphere energy cycle. Mon. Wea. Rev., v.92, p.483-493, 1964.

PEREZ-LANDA, G.; CIAIS, P.; SANZ, M. J.; GIOLI, B.; MIGLIETTA, F.; PALAU, J. L.; GANGOITI, G.; MILL'NA, M. M.; Mesoscale circulations over complex terrain in the 
Valencia coastal region, Spain - Part 1: Simulation of diurnal circulation regimes. Atmos. Chem. Phys., 7, 1835-1849, 2007.

PIELKE, R. A; Mesoscale Meteorological Modeling. $2^{\mathrm{a}}$ ed., Academic Press, 2002. p. $462-468$.

PLANCHON, O.; DAMATO, F.; DUBREUIL, V.; GOUERY, P.; A method of identifying and locating sea-breeze fronts in north-eastern Brazil by remote sensing. Meteorol. Appl., 13, 225-234, 2006.

PORSON, A.; STEYN, D. G.; SCHAYES, G.; Sea-breeze scaling from numerical model simulations, part II: Interaction between the sea breeze and slope flows. Boundary-Layer Meteorol., 122, 31-41, 2007.

RENNÓ, N. O.; BLUESTEIN, H. B.; A simple theory for waterspouts. J. Atmos. Sci. v.58, p.927-932. 2001.

RENNÓ, N. O.; BURKETT, M. L.; LARKIN, M. P.; A simple theory for dust devils. J. Atmos. Sci, v.55, p.32244-3252, 1998.

RENNÓ, N. O.; INGERSOLL, A. P.; Natural convection as a heat engine: A theory for CAPE. J. Atmos. Sci., v.53, p.572-585, 1996.
RIEHL, H.; A model of hurricane formation. J. Appl. Phys., v.21, p.917-925, 1950.

SMAGORINSKY, J.; General circulation experiments with the primitive equations. Part I: The basic experiment. Mon. Wea. Rev. v. 91, p. 99-164, 1963.

SOUZA, E. P.; Estudo teórico e numérico entre convecção e superfícies heterogêneas na Região Amazônica. TESE DE DOUTORADO. Universidade de São Paulo, 7-31, 1999.

SOUZA, E. P.; Trabalho de expansão e de compressão associado às circulações convectivas. Revista Brasileira de Meteorologia, v. 19, n. 2, p. 141-148. 2004.

SOUZA, E. P.; RENNÓ, N. O.; SILVA DIAS, M. A. F.; Convective circulations induced by surface heterogeneities. J. Atmos. Sci., v.57, p.2915-2922, 2000.

WALKO, R.; COTTON, W.R.; MEYERS, M.P.; HARRINGTON, J.Y.; New RAMS cloud microphysics parameterization. Part I: The single-moment scheme. Atmos. Res. V. 38, p. 29-62, 1995.

WILLIAMS, E.; RENNÓ, N.; An analysis of the conditional instability of the tropical atmosphere. Mon. Wea. Rev. v. 121, p. 21-36, 1993. 